\title{
Effect of nicotine-caffeine interaction on rats coronaries and cardiac muscle
}

\author{
Soad Shaker Ali ${ }^{1}$, Mohamed Nabil Alama ${ }^{2}$, Etedal Abbas Huwait ${ }^{3}$, Amal Saeed Abed ${ }^{4}$, and Gamal Karrouf ${ }^{* 5,6}$ \\ ${ }^{1}$ Department of Anatomy (Cytology and Histology), Faculty of Medicine, King Abdulaziz University, Jeddah 21589, Saudi Arabia and Assuit University, Faculty of \\ Medicine, Histology Department, Egypt \\ ${ }^{2}$ Consultant Adult Interventional Cardiologist, King Abdulaziz Hospital, King Abdulaziz University, Jeddah 21589, Saudi Arabia \\ ${ }^{3}$ Department of Biology, Faculty of Science, King Abdulaziz University, Jeddah 21589, Saudi Arabia \\ ${ }^{4}$ Master Technical Anatomy, Department of Anatomy (Cytology and Histology), Faculty of Medicine, King Abdulaziz University, Jeddah 21589, Saudi Arabia \\ ${ }^{5}$ Faculty of Science, Medical Physics Department, King Abdulaziz University, Jeddah 21589, Saudi Arabia \\ ${ }^{6}$ Surgery, Anesthesiology and Radiology Department, Faculty of Veterinary Medicine, Mansoura University, Mansoura, 35516 Dakahlia, Egypt
}

\begin{abstract}
The influence of nicotine and caffeine interaction at dose levels approximating human consumption on histology of cardiac muscles and coronaries was investigated in rats. Adult male rats were sorted into four groups, GI: control and injected subcutaneously with 2 ml normal saline. GII: received subcutaneous nicotine injection in a dose of $(10 \mathrm{mg} / \mathrm{kg} /$ body weight). GIII: received daily intravenous injection of caffeine (100 mg/ $\mathrm{kg})$. GIV: received a combination of nicotine and caffeine in similar dose and route. Nicotine leads to marked congestion, damage in cardiac muscles, increase in collagen fibers around thickened coronaries. The effect of caffeine produced little change and congestion in cardiac fibers and slight increase in collagen fibers around coronaries. In group 4 caffeine prevent the increase in collagen fibers around coronaries but did not protect cardiac muscles against congestion and damage by nicotine. The present study indicated that nicotine causing structural damage and changes in the histological profile of the cardiac muscles and coronaries of rats and caffeine could modify nicotine effect on coronary walls but ischemic changes in cardiac muscles seemed to be irreversible.
\end{abstract}

\section{Introduction}

Nicotine and caffeine represent two of the most common pharmacologically active substances used by human. These are responsible for $80 \%$ of all diagnoses of cerebrovascular and coronary heart diseases [1-3]. Coronary endothelial dysfunction and development of atherosclerotic changes and risk of cardiac complication and deaths were reported in heavy smokers [4,5]. Caffeine is classified as an addictive substance and consumed all over the world to relieve sense of fatigue, improve mood concentration, insomnia, and increased heart rate [6-8]. This study aimed to document the effect of nicotine-caffeine interaction on histology of coronaries and cardiac muscle of adult male rats.

\section{Materials and methods}

\section{Pharmacological agents}

Nicotine tartrate and caffeine were obtained from Sigma Chemical Co. , Jeddah, Saudi Arabia.

\section{Animal treatment}

Animals and experimental design: The present study was conducted at King Fahd Medical Research Center, King Abdulaziz University, and Jeddah, Saudi Arabia on a total number of 24 apparently healthy albino male rats, weighted from 200 to 250 grams. Rats had free access to commercial rat pellets and tap water for one week before experiment. The study was performed according to animal care ethics recommended by the University Committee. These animals were allocated into4 groups $(\mathrm{n}=6)$ as follow:

Group I: control and injected subcutaneously with $2 \mathrm{ml}$ normal saline.
Group II: Nicotine group received daily subcutaneous nicotine/ saline $(10 \mathrm{mg} / \mathrm{kg} /$ body weight.

Group III: caffeine treated group (received daily intrapertioneally caffeine anhydrous $100 \mathrm{mg} / \mathrm{kg} /$ body weight).

Group IV: caffeine and nicotine treated groups given in the same doses and by thesame routes as GI \& GII. After 4 weeks animals were euthanized by cervical dislocation under deep ether anesthesia. The heart was perfused with normal saline followed by $10 \%$ neutral buffered formalin. Cross sections from left ventricles at different levels from the apex were re-fixed in $10 \%$ neutral buffered formalin for paraffin processing. 5 micron thick sections were stained by haematoxylin and eosin for general structure. Masson Trichrome was used for collagen fibers. Slides prepared from all groups were examined and photographed [9].

\section{Results}

\section{Effect on coronaries}

In GI (control group) showed that rat cardiac muscles of left

Correspondence to: Prof. Gamal Karrouf, Faculty of Science, Medical Physics Department, King Abdulaziz University, 21589 Jeddah, Saudi Arabia and Surgery, Anesthesiology and Radiology Department, Faculty of Veterinary Medicine, Mansoura University, Mansoura, 35516 Dakahlia, Egypt

Key words: nicotine, caffeine, coronary, cardiac muscles, rats

Received: January 20, 2017; Accepted: February 28, 2017; Published: March 03, 2017 
ventricle possess normal structure described in literature. The nuclei of muscles are oval and vesicular. The fiber cytoplasm is homogenously stained but the cross striation are ill defined. Capillaries among the fibers are thin wall and compressed. Coronaries showed normal ordered layers. The inner intimal layer showed smooth intact endothelial cells Media was mainly made of elastic fibers with few smooth muscles and collagen fibers. Those two layers' merge together to make one intimamedia layer. Adventitia or the most outer layer consisted from loose collagen fibers and perivascular tissue is scanty and contains thin fibers and few connective tissue cells. Caffeine on the other hand produced no change in coronary wall thickness. Slight capillary congestion was observed but cardiac muscles did not show any histological changes. In animals administrated combination of nicotine and caffeine, there was no change in coronary wall thickness but cardiac fibers still showed histological changes observed in nicotine group (Figure 1).

\section{Masson stain and collagen}

Masson trichrome stain was used to demonstrate the changes in collagen content around coronaries and any fibrotic changes in cardiac muscles. Nicotine leads to increase in collagen fibers around thickened coronaries. The effect of caffeine showed slight increase in collagen fibers around coronaries. In group 4 caffeine prevent the increase in collagen fibers around coronaries (Figure 2).

\section{Effect on cardiac muscle}

Longitudinal section (LS) of cardiac muscles of controlled rats (GI) showed normal cardiac fibers. Nicotine (GII) showed shrinkage and irregularity of cardiac fibers, caffeine(GIII): produced slight shrinkage in cardiac fibers with mild congestion of interfibrillar capillaries, combination of nicotine and caffeine (G1V) showed that caffeine not protect against nicotine induced histological cardiac changes (Figure
3). Cross section (CS) of cardiac muscles show GI showed regular and normal sized cardiac fibers .GII (nicotine) showed marked vascular congestion( white stars).nearby muscles looked darker and degenerated (black stars) GIII (caffeine) showed no apparent changes in cardiac fibers, while administration of Caffeine in combined with nicotine (GIV) did not protect cardiac muscle from nicotine effect (Figure 4).

\section{Discussion}

The smoking effects on human health are well known and its impact on vital organs and functions can leads to deaths in many cases especially when was accompanied by cardiovascular complication. Cardiac muscles with their coronaries were the most involved parts [10]. Few studies have examined the effects of tobacco on cardiac muscles and coronaries. The results of this research declared that nicotine have deleterious effects on the heart, and coronaries and structural damage to the histological profile It result in increase in coronary wall thickness and increase perivascular type I collagen deposition as observed by Masson trichrome stain. Cardiac muscles showed numerous foci of apoptotic fibers $[11,12]$.

Damage of coronaries and cardiac muscles could be induced via free radicles and oxidative stress known to be induced by nicotine. Nicotine was known to result in sympathetic neural stimulation and systemic catecholamine release [13]. Czernin and Waldherr [14] reported an increase liberation of reactive oxygen upon exposure to nicotine with subsequent injury of vascular endothelium decrease coronary blood flow, increased cardiac metabolic demand and increase risk of myocardial ischemic changes and initiation of atherosclerotic changes. Based on such data one can explain the degenerative apoptotic changes in cardiac observed in nicotine injected rats.

In the present study caffeine administration on the other hand produced no change in coronary wall thickness. or did not alter its

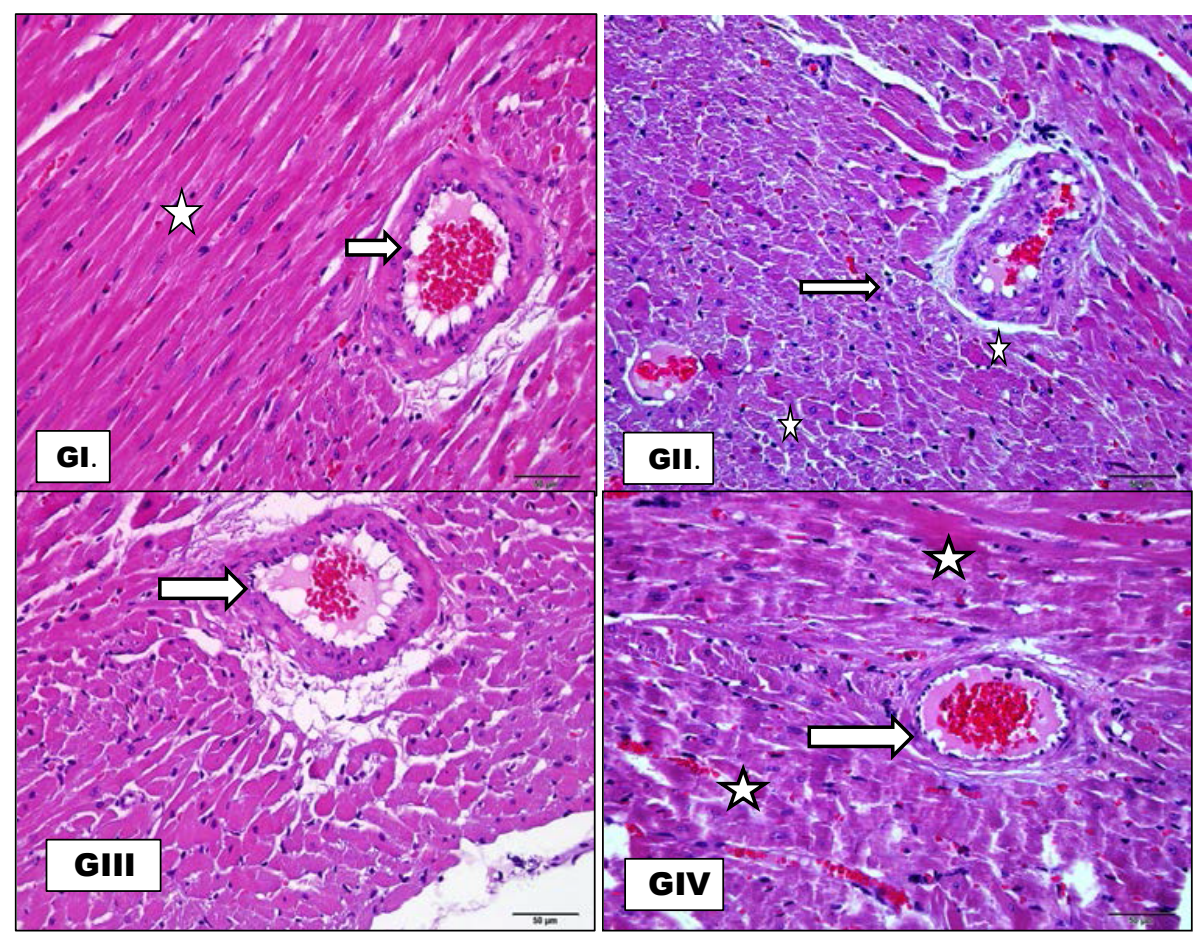

Figure 1. Cross section of coronaries, G1: control with normal wall thickness (white arrow) and well organized nearby cardiac muscle fibers ( star) .GII:(nicotine) showing increased wall thickness (white arrows). Nearby cardiac fibers (thin black arrows) showed apoptotic changes (dark red shrunken fibers with dark pyknotic nuclei) or tiny lipid droplets (white stars). GIII: Caffeine produced no change in coronary wall thickness (white arrow) or cardiac fibers. GIV: Caffeine combined with nicotine protected coronary from the effect of nicotine but still some degeneration in cardiac muscles (White stars) (H\&E x20). 


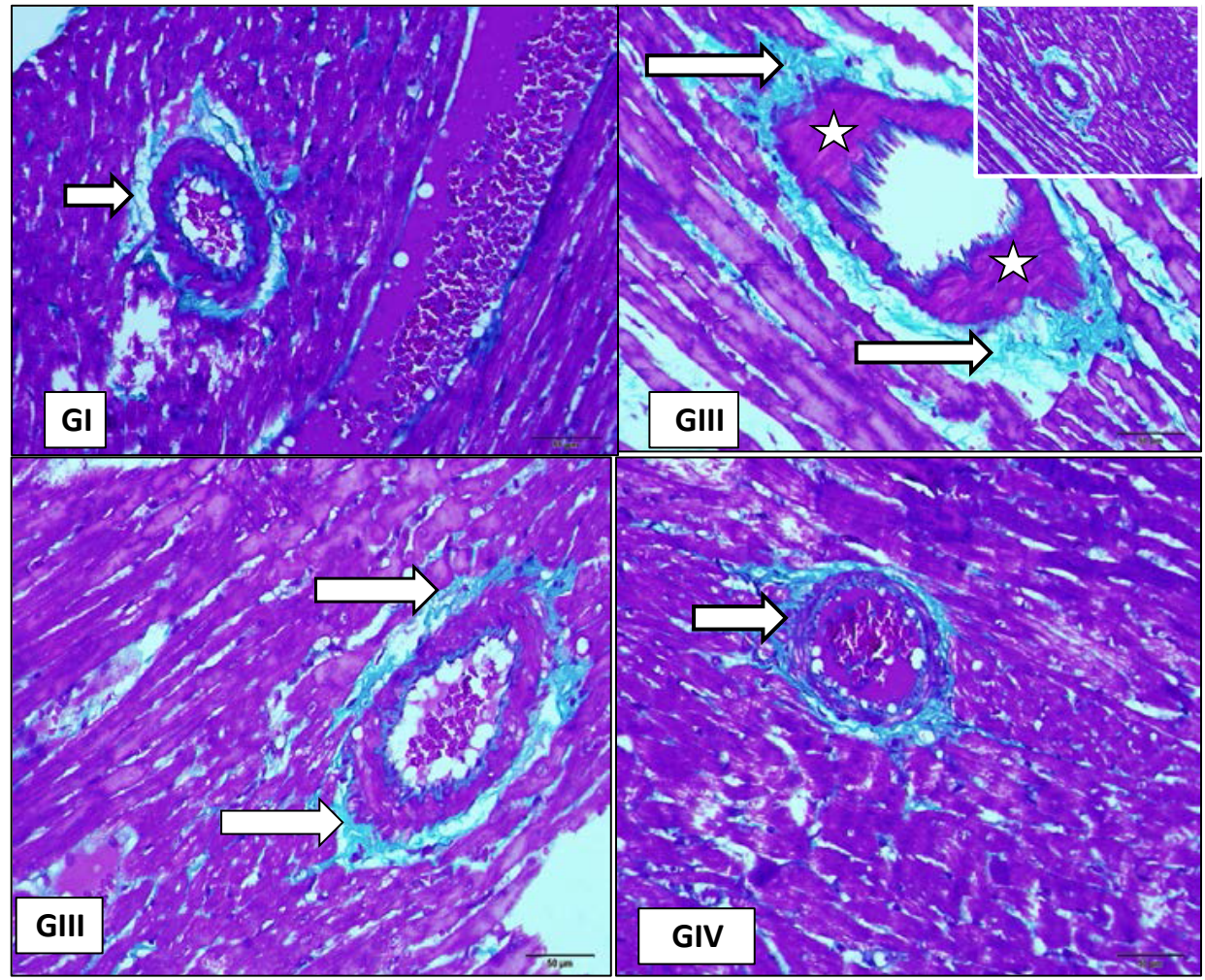

Figure 2. Cross section in coronaries G1: control with normal wall thickness (white arrow), GII: Nicotine leads to increased wall thickness (white stars) and increase in collagen fibers around thickened coronaries ( white arrows). GIII: caffeine result in slight increase in collagen fibers around coronaries (white arrows), GIV: Caffeine combined with nicotine prevents the increase in collagen fibers around coronaries (Masson Trichrome).
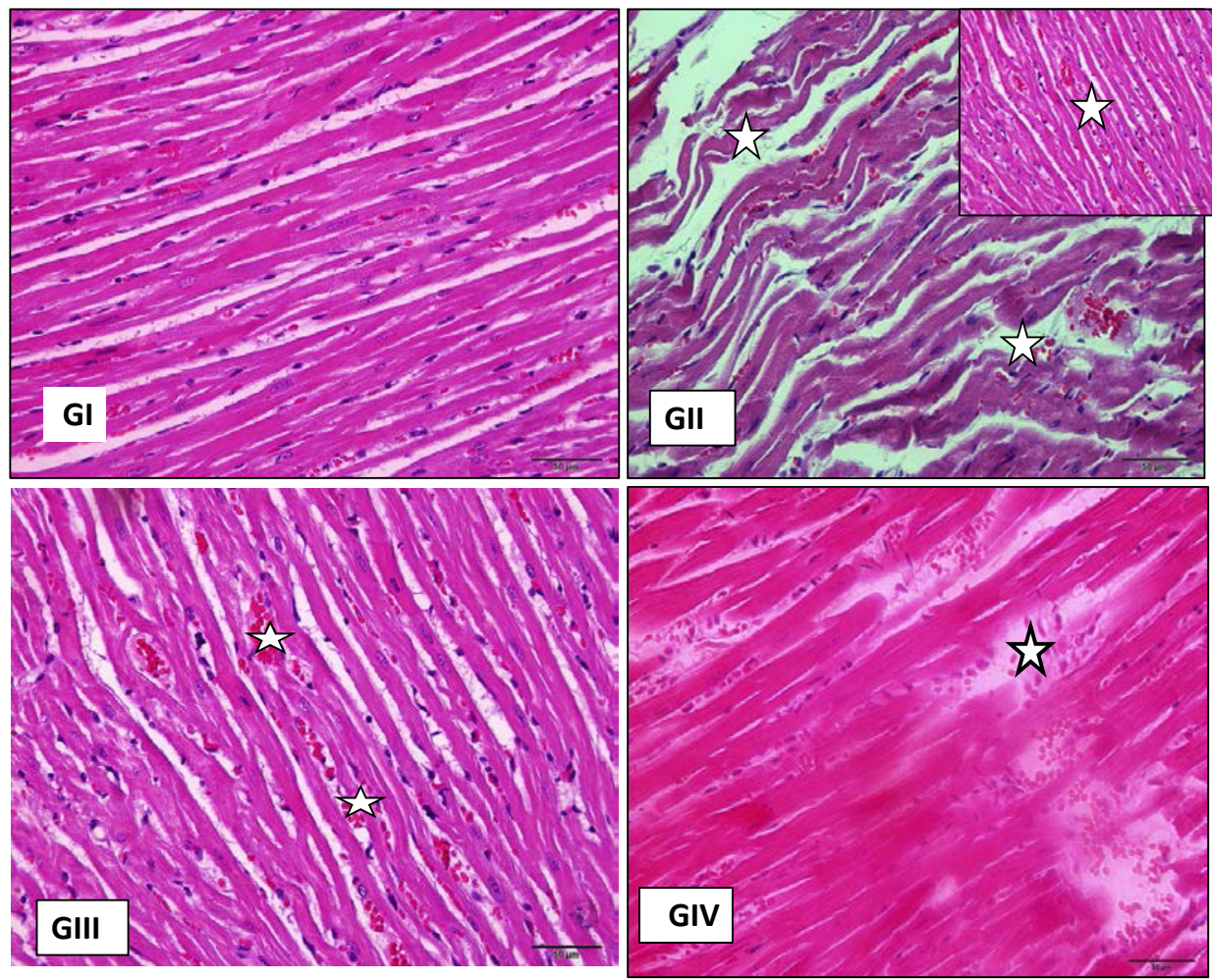

Figure 3. LS in cardiac muscles Showed: G1(control): normal cardiac fibers. GII: (nicotine)showed shrinkage and irregularity of cardiac fibers. (GIII): caffeine produced slight shrinkage in cardiac fibers with mild congestion of interfibrillar capillaries (white starts) G1V: combination of nicotine andcaffeine showed that caffeine not protectagainst nicotine induced histological cardiac changes, (H\&E stain x20). 


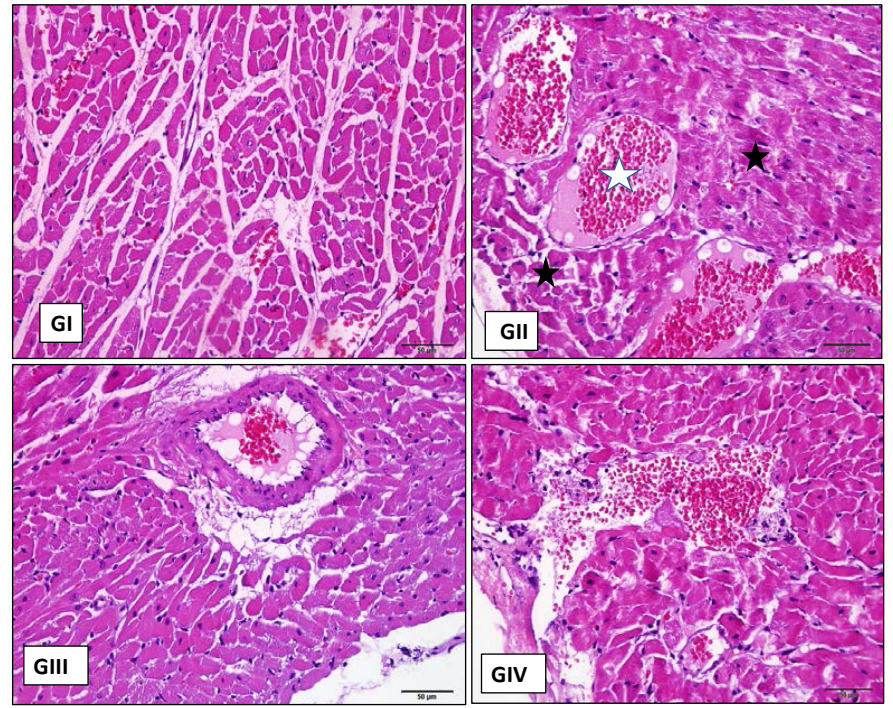

Figure 4. Cross section to show GI: control with regular control and normal sized cardiac fibers .GII : ( nicotine) showed marked vascular congestion (white stars) nearby muscles looked darker and degenerated ( black stars) GIII : caffeine with no apparent changes in cardiac fibers. GIV: administration of caffeine in combined with nicotine didnot protect cardiac muscle from nicotine effect (H\&E stain x20).

structure, but it resulted in dilation of cardiac interfibrillar capillaries with no apparent histopathological changes [15,16]. Caffeine consumption when combined with nicotine is considered stimulants. In the present study, administration of caffeine together with nicotine result in preservation of coronary wall thickness but cardiac fibers still showed histological changes observed in nicotine group [17]. Higgins and Babu [18] demonstrated increased 10 percent in resting flow-mediated dilatation inside brachial artery after caffeine ingestion. Caffeine's effect on blocking adenosine-induced vasodilatation in the coronary arteries in normal healthy subjects.

Our findings suggest that nicotine leads to marked vascular congestion nearby muscles looked darker and degenerated while caffeine showed no apparent changes in cardiac fibers and sudden deaths were not recorded [13] while Nyska et al. [15] demonstrated acute hemorrhagic myocardial necrosis and sudden death of rats exposed to a combination of ephedrine and caffeine.

\section{Conclusion:}

Caffeine could modify nicotine effect on coronary walls but ischemic changes in cardiac muscles seemed to be irreversible.

\section{References}

1. Kobayashi Y, Takeuchi T, Hosoi T, Loeppky JA (2004) Effects of habitual smoking on cardiorespiratory responses to sub-maximal exercise. J Physiol Anthropol Appl Human Sci. 23: 163-169.

2. Bullen C (2008) Impact of tobacco smoking and smoking cessation on cardiovascular risk and disease. Expert Rev Cardiovasc Ther 6: 883-895. [Crossref]

3. Ubhi HK, Kotz D, Michie S, van Schayck OC, Sheard D, et al. (2016) Comparative analysis of smoking cessation smartphone applications available in 2012 versus 2014. Addict Behav 58: 175-181. [Crossref]

4. Kotz D, Brown J, West R (2014) Prospective cohort study of the effectiveness of varenicline versus nicotine replacement therapy for smoking cessation in the "real world". BMC Public Health 14: 1163.

5. Liu CC, Yeh HI (2014) Nicotine: A Double-Edged Sword in Atherosclerotic Disease. Acta Cardiol Sin 30: 108-113. [Crossref]

6. Battram DS, Shearer J, Robinson D, Graham TE (2004) Caffeine ingestion does not impede the resynthesis of proglycogen and macroglycogen after prolonged exercise and carbohydrate supplementation in humans. J Appl Physiol 10: 943-950.
7. Howden R. Hanlon PR. Petranka JG. Kleeberger S. Bucher J, et al. (2005) Ephedrine plus caffeine causes age-dependent cardiovascular responses in Fischer 344 rats. Am J Physiol Heart Circ Physiol 288: H2219-H2224.

8. Gibbs BF, Gonçalves Silva I, Prokhorov A, Abooali M, Yasinska IM, et al. (2015) Caffeine affects the biological responses of human hematopoietic cells of myeloid lineage via down regulation of the mTOR pathway and xanthine oxidase activity Oncotarget 30: 28678-28692.

9. Bancroft JD, Gamble M (2008) Theory and practice of histological techniques, 6th edn Churchill Livingstone, Philadelphia.

10. Czernin J, Waldherr C (2003) Cigarette smoking and coronary blood flow. Prog Cardiovasc Dis 45: 395-404. [Crossref]

11. Ito U, Spatz M, Walker JT Jr, Klatzo I (1975) Experimental cerebral ischemia in mongolian gerbils. I. Light microscopic observations. Acta Neuropathol 32: 209-223. [Crossref]

12. Adedayo AD, TijaniAA, MusaAA, AdeniyiTD (2011) Histological study of smoke extract of Tobacco nicotiana on the heart, liver, lungs, kidney, and testes of male Sprague-Dawley rats. Niger Med J 52: 217-222.

13. Adamopoulos D, van de Borne P, Argacha JF (2008) New insights into the sympathetic, endothelial and coronary effects of nicotine. Clin Exp Pharmacol Physiol 35: 458-463. [Crossref]

14. Nyska A, Murphy E, Foley JF, Collins BJ, Petranka J, Howden R, Hanlon P, Dunnick JK (2005) Acute hemorrhagic myocardial necrosis and sudden death of rats exposed to a combination of ephedrine and caffeine. Toxicol Sci 83: 388-396

15. Brown CE, Stanley Trauth E, Richard GrippoS, GurleyBill J and GrippoAnne A (2012) Combined Effects of Ephedrine-Containing Dietary Supplements, Caffeine, and Nicotine on Morphology and Ultrastructure of Rat Hearts. J Caffeine Res 2: 123-132.

16. Dunnick JK, Kissling G, Gerken DK, Vallant MA, Nyska A (2007) Cardiotoxicity of Ma Huang/caffeine or ephedrine/caffeine in a rodent model system. Toxicol Pathol 35 657-664. [Crossref]

17. Higgins JP, Babu KM (2013) Caffeine reduces myocardial blood flow during exercise. Am J Med 126: 730. [Crossref]

18. Nurkiewicz TR1, Porter DW, Barger M, Castranova V, Boegehold MA (2004) Particulate matter exposure impairs systemic microvascular endothelium-dependent dilation. Environ Health Perspect 112: 1299-1306. [Crossref]

Copyright: (C2017 Ali SS. This is an open-access article distributed under the terms of the Creative Commons Attribution License, which permits unrestricted use, distribution, and reproduction in any medium, provided the original author and source are credited. 